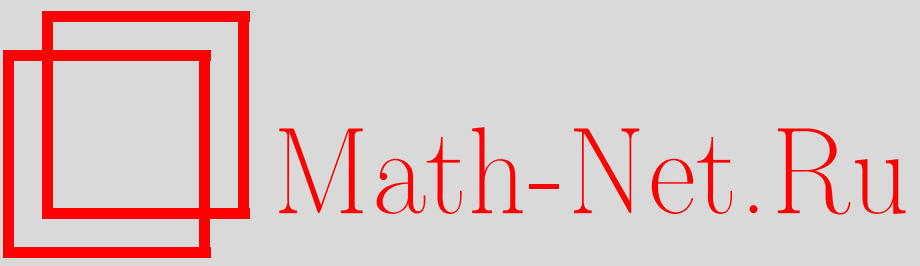

С. В. Людковский, Меры на группах диффеоморфизмов неархимедовых многообразий, представления групп и их применения, ТМФ, 1999, том 119, номер 3, 381-396

DOI: https://doi.org/10.4213/tmf746

Использование Общероссийского математического портала Math-Net.Ru подразумевает, что вы прочитали и согласны с пользовательским соглашением

http://www. mathnet.ru/rus/agreement

Параметры загрузки:

IP : 54.157 .27 .8

26 апреля 2023 г., 14:10:25 
ТЕОРЕТИЧЕСКАЯ

И МАТЕМАТИЧЕСКАЯ

ФИЗИКА

Том 119, № 3

июнь, 1999

(C) $\mathbf{1 9 9 9}$ г.

С.В. Людковский*

\section{МЕРЫ НА ГРУППАХ ДИФФЕОМОРФИЗМОВ НЕАРХИМЕДОВЫХ МНОГООБРАЗИЙ, ПРЕДСТАВЛЕНИЯ ГРУПП И ИХ ПРИМЕНЕНИЯ}

Построены невырожденные $\sigma$-аддитивные квазиинвариантные и псевдодифференцируемые относительно плотных подгрупп $G^{\prime}$ меры со значениями в $\mathbf{R}$ и $\mathbf{Q}_{q}$ для простых $q \neq p$ на группах диффеоморфизмов $G$ и гомеоморфизмов неархимедовых банаховых сепарабельных многообразий $M$ над локальным полем $\mathbf{K}$, где $\mathbf{Q} p$ - поле $p$-адических чисел, $\mathbf{K} \supset \mathbf{Q}_{p}$. Меры и ассоциированные неприводимые представления использованы в теории неархимедовой гравитации.

\section{1. ВВЕДЕНИЕ}

Группы диффеоморфизмов и их неприводимые представления играют важную роль в квантовой механике и теории квантовой гравитации [1]. В последнее время развиваются также неархимедовы квантовая механика и квантовая гравитация $[2,3]$.

В данной статье в разделах 2 и 3 построены меры. В п. 3.7 исследована неприводимость регулярных унитарных представлений группы диффеоморфизмов $G^{\prime}$. В разделе 4 приводятся примеры применения таких представлений в неархимедовых квантовой механике и квантовой гравитации.

\section{2. СПЕЦИФИЧЕСКИЕ ИЗОМОРФИЗМЫ ПРОСТРАНСТВ}

2.1. Обозначим через $C\left(\operatorname{an}_{r}, M \rightarrow Y\right)$ пространство аналитических отображений $g: M \rightarrow Y$ с радиусом сходимости не меньше чем $0<r<\infty$, где $M$ открыто-замкнуто в $\mathbf{B}(X, 0, r), X$ и $Y$-банаховыпространства (БП) над $\mathbf{K}, \mathbf{B}(E, x, R):=\{y \in E: d(y, x) \leqslant$ $R\}$ - шар для метрического пространства $(E, d)$.

2.2.1. Возьмем последовательность $\left\{H(n): n \in \mathbf{N}_{0}\right\}$ БП над полем $\mathbf{K}$, где $\mathbf{N}_{0}:=$ $\{0,1,2, \ldots\}, \mathbf{N}:=\{1,2, \ldots\}$. Зададим БП

$$
\begin{gathered}
c_{0}(H(n): n):=\left\{f=\left(f^{0}, f^{1}, f^{2}, \ldots\right): \quad f^{n} \in H(n) \text { для любых } n\right. \text { и } \\
\left.\left\|f^{n}\right\|_{H(n)} \rightarrow 0 \text { при } n \rightarrow \infty\right\}
\end{gathered}
$$

* Институт общей физики РАН, Москва, Россия 
с нормой

$$
\|f\|_{c_{0}(H(n): n)}:=\sup _{n}\left\|f^{n}\right\|_{H(n)} .
$$

Пусть $X$ - БП сепарабельного типа над полем $\mathbf{K}$, тогда оно изоморфно БП $c_{0}(\alpha, \mathbf{K}):=$ $c_{0}(\mathbf{K}: n)$ при $\alpha=\omega_{0}, c_{0}(\alpha, \mathbf{K})=\mathbf{K}^{\alpha}$ при $\alpha<\omega_{0}$, где $\alpha$ - ординал, $\alpha \leqslant \omega_{0}, \omega_{0}$-начальный ординал мошности $\aleph_{0}:=\operatorname{card}(\mathbf{N}), \operatorname{dim}_{\mathbf{K}} X:=\operatorname{card}(\alpha)$, БП $X$ имеет стандартный ортонормированньй базис $\left(e_{j}: j \in \alpha\right)[4]$.

Здесь и далее рассматриваются аналитические банаховы многообразия (БМ) $M$ и $N$, моделируемые на $X$ и $Y$ с атласами $\operatorname{At}(M)$ и $\operatorname{At}(N)$, состоящими из дизъюнктных карт $\left(U_{j}, \phi_{j}\right), \quad j \in \Lambda ; \quad\left(V_{j}, \psi_{j}\right), \quad j \in \Omega, \Omega \cup \Lambda \subset \mathbf{N}$, где $U_{j}, \phi_{j}\left(U_{j}\right), \quad V_{j}$ и $\psi_{j}\left(V_{j}\right)$ открыто-замкнуты в $M, X, N$ и $Y$, соответственно, $\phi_{j}: U_{j} \rightarrow \phi_{j}\left(U_{j}\right)$ и $\psi_{j}: V_{j} \rightarrow \psi_{j}\left(V_{j}\right)-$ гомеоморфизмы, а $\phi_{j}\left(U_{j}\right)=\mathbf{B}\left(X, x_{j}, r_{j}\right)$ с $0<r_{j}<\infty$ для любых $j$.

2.2.2. Для $\Lambda=\omega_{0}$ определим БП

$$
\begin{aligned}
C_{*}(t, M \rightarrow Y):= & \left\{\left.f\right|_{U_{j}} \in C_{*}\left(t, U_{j} \rightarrow Y\right),\|f\|_{C_{*}(t, M \rightarrow Y)}:=\right. \\
:= & \sup _{j \in \Lambda}\left(\left\|\left.f\right|_{U_{j}}\right\|_{C_{*}\left(t, U_{j} \rightarrow Y\right)} / \min \left(1, r_{j}\right)\right)<\infty \text { и } \\
& \left.\left(\left\|\left.f\right|_{U_{j}}\right\|_{C_{*}\left(t, U_{j} \rightarrow Y\right)} / \min \left(1, r_{j}\right)\right) \rightarrow 0 \text { при } j \rightarrow \infty\right\},
\end{aligned}
$$

где $0 \leqslant t<\infty, *=0$ для пространств $C_{0}(t, U \rightarrow Y), *=\varnothing$ или просто опускается для $C(t, U \rightarrow Y)$ (о $C(t, M \rightarrow Y)$ в случае конечного $\operatorname{At}(M)$ см. также [5, § 2] и [6]). Через $C_{*}^{\theta}(t, M \rightarrow N)$ при $0 \leqslant t \leqslant \infty$ обозначим пространство функций $f: M \rightarrow N$ таких, что $\left(f_{i}-\theta_{i}\right) \in C_{*}(t, M \rightarrow Y)$ для любых $i \in \Omega$ и $f_{i}=\psi_{i} \circ f, \theta_{i}=\psi_{i} \circ \theta$; аналогично для $C_{*}((t, s) ; M \rightarrow N)\left(\right.$ см. [7]) при $\operatorname{dim}_{\mathbf{K}} M=n \in \mathbf{N}$. Введем группы

$$
G(\tau, M):=C_{0}^{\mathrm{id}}(\tau, M \rightarrow M) \cap \operatorname{Hom}(M),
$$

которые называются группами диффеоморфизмов (и гомеоморфизмов при $0 \leqslant t<1$ и $s=0)$, где $\operatorname{Hom}(M)$ - группа непрерьвных гомеоморфизмов, $\tau=(t, s)$ или $\tau=\operatorname{an}_{r}, s=$ 0 опускается.

2.3. Для $\mathbf{B}(X, 0,1)=: \mathbf{B}$ положим $\widetilde{\mathbf{B}}:=\left\{x=\left(x^{i}: i \in \alpha\right) \in \mathbf{B}:\right.$ существует $i \in \alpha \mathrm{c}$ $x^{i}=0$ или $\left.x^{i}=1\right\}$. Для каждого $\infty>r>0$ c $r \in \Gamma_{\mathbf{K}}:=\left\{|x|_{\mathbf{K}}: 0 \neq x \in \mathbf{K}\right\}$ фиксируем $\xi_{r} \in \mathbf{K}$ с $\left|\xi_{r}\right|_{\mathbf{K}}=r$, определяем $\widetilde{\mathbf{B}}\left(X, y_{i}, r\right):=y_{i}+\widetilde{\mathbf{B}}(X, 0,1) \times \xi_{r}$, где выбрано фиксированное множество $\left(y_{i}: i \in \mathbf{N}\right)$ для $r$ такое, что $y_{1}=0$ и $X$ равно дизъюнктному объединению шаров $\mathbf{B}\left(X, y_{i}, r\right)$ и полагаем

$$
\widetilde{M}:=\bigcup_{j} \phi_{j}^{-1}\left(\widetilde{\mathbf{B}}_{j}\right)
$$

(см. [5] и п. 2.2.1).

Для локально-компактного $M$ зададим подпространство и подгруппу

$$
\begin{aligned}
C\left(t, M \rightarrow \mathbf{K}^{a} \mid h\right) & :=\left\{f \in C\left(t, M \rightarrow \mathbf{K}^{a}\right):\left.\Delta^{j} f\right|_{\widetilde{M}}=0 \quad \forall j=0, \ldots, v(h, n)\right\}, \\
\operatorname{Dif}(t, M \mid h) & :=\left\{f \in G(t, M):(f-\mathrm{id}) \in C\left(t, M \rightarrow \mathbf{K}^{n} \mid h\right)\right\},
\end{aligned}
$$


где $v(h, n)=2 h-1$ для четного $l^{\prime}, v(h, n)=2 h$ для нечетного $l^{\prime}, s \geqslant 2, l^{\prime}$ и $s \in \mathbf{N}$,

$$
h=\left[\frac{l^{\prime}}{2}\right], \quad l \geqslant l^{\prime} \geqslant s n, \quad \Delta^{0}:=1, \quad \partial_{j}:=\frac{\partial}{\partial x^{j}}, \quad \Delta=\Delta_{n}=\partial_{1}^{2}+\cdots+\partial_{n}^{2}
$$

- лапласиан, $n:=\operatorname{dim}_{\mathbf{K}} M ;[t]=: l \leqslant t \in \mathbf{R}$ либо $t=\operatorname{an}_{R}$.

2.4. Пусть $u \geqslant p$ и $s>1$ - целые числа. Тогда система линейных алгебраических уравнений

$$
\begin{array}{r}
\{c(d, d, w) \chi(d, u)+\cdots+c(p, d, w) \chi(p, u)=0 \quad \text { для } \quad d=0, \ldots, w-1 \\
\text { с } w=1, \ldots, \min (s-1, u)=: p \quad \text { и } \quad \chi(0, u)+\cdots+\chi(p, u)=1\}
\end{array}
$$

имеет единственное решение с $\chi(j, u) \in \mathbf{Q}($ см. $[8,9])$, где

$$
c(j, d, w):=\frac{(u-j) ! j !}{(w-d) !(u-j-w+d) !(j-d) ! d !} .
$$

2.5.1. Пусть $F$ - дифференциальный оператор, действующий на $f \in C_{0}\left(t, M \rightarrow \mathbf{K}^{n}\right)$, тогда сушествует оператор $[F]$, в котором все $\partial_{i}$ заменены операторами разделенных разностей $\left(\bar{\Phi}^{1} f\right)\left(x ; e_{i} ; \zeta\right)[5]$.

2.5.2. Для локально-компактного $M$ сушествует БП

$$
E\left((t, s),-k, N \rightarrow \mathbf{K}^{n}\right)=[\tilde{A}] C_{0}\left((t, s), M \rightarrow \mathbf{K}^{n}\right)
$$

с нормой

$$
\|g(y)\|_{E}:=\sup _{i, j, m}\left\{\left|a\left(m,\left.f^{i}\right|_{U_{j}}\right)\right| \frac{J_{j}((t, s), m)}{J_{j}(k, m) \min \left(1, r_{j}\right)}\right\}
$$

где $g(y)=([\tilde{A}] f)(y), y \in N=M^{k+1}, y^{1}=x, y^{i+1}=x+\zeta_{i} h_{i}$ при $i>0, y=\left(y^{1}, y^{2}, \ldots\right)$, $\zeta_{i} \in \mathbf{K}, h_{i} \in X, k=2 q=\operatorname{deg}[\tilde{A}], \tilde{A}=\Delta^{q}, J_{j}((t, 0), m)=: J_{j}(t, m), \quad s \in \mathbf{N}_{0}$, индекс $s=0$ далее опускаем.

2.5.3. Пусть $W_{\mathrm{id}}-$ окрестность id в $G(t, M)$ с $t \geqslant 1$ такая, что $\left(W_{\mathrm{id}}-\mathrm{id}\right) \subset C_{0}(t$, $M \rightarrow X)$ (см. также раздел I.3.5(iv) в [8]). Тогда на $W_{\mathrm{id}}$ можно задать операторы

$$
A_{n}(f)=\sum_{(i=(i(1), \ldots, i(n)),|i|=s n)}\left[\frac{(s n) !}{i(1) ! \ldots i(n) !}\right] A\left(i(1), \ldots, i(n) ; f^{\prime}, f\right)
$$

и

$$
A\left(i ; f^{\prime}, f\right)=\partial_{1}^{2 i(1)} \ldots \partial_{r-1}^{2 i(r-1)} \partial_{r+1}^{2 i(r+1)} \ldots \partial_{n}^{2 i(n)} \sum_{l=0}^{s-1} \chi(l, 2 i(r)) \partial_{r}^{l}\left[\left(f^{\prime}\right)^{-1} \partial_{r}^{2 i(r)-l} f\right]
$$

где $s \geqslant 2, \tau \geqslant s n, r=\min \{j: i(j)>s-1\}$ (см. п. 2.4).

2.5.4. Для $C_{0}\left((t, s), M \rightarrow \mathbf{K}^{n} \mid h\right) \ni f$ с помощью алгоритма исключения Гаусса получаются выражения зависимых коэффициентов разложений $a\left(m,\left.f^{i}\right|_{U_{j}}\right)$ функций 
$f$ по базису $\left(\left.\bar{Q}_{m}\right|_{U_{j}}\right) q_{i}$ (выбранных среди $a\left(m, f^{i}\right)$ с $m(k) \leqslant 2 v(h, n)$ для некоторого $k \in\{1, \ldots, n\})$ через независимые $a\left(m,\left.f^{i}\right|_{U_{j}}\right)$. Исходя из этого получим изоморфизмы

$$
\begin{aligned}
\widetilde{T}_{C}^{(t, s)} & : C_{0}\left((t, s), M \rightarrow \mathbf{K}^{n}\right) \rightarrow C_{0}\left(\operatorname{an}_{r}, M \rightarrow \mathbf{K}^{n} \mid h\right), \\
T_{E}^{(t, s, k)}: & :\left((t, s),-k, M^{k+1} \rightarrow \mathbf{K}^{n}\right) \rightarrow E\left(\operatorname{an}_{r},-k, M^{k+1} \rightarrow \mathbf{K}^{n}\right) .
\end{aligned}
$$

В окрестности $W_{\text {id }}$ в $\operatorname{Dif}((t, s), M \mid h)$ или $G((t, s), M), f \in W_{\text {id }}$, рассмотрим операторы

$$
T f:=\mathrm{id}+T_{C}^{(t, s)}(f-\mathrm{id}) \quad \text { или } \tilde{T} f:=\mathrm{id}+\widetilde{T}_{C}^{(t, s)}(f-\mathrm{id}),
$$

а для обратного оператора $S_{E}^{(t, s, k)}=\left(T_{E}^{(t, s, k)}\right)^{-1}$ рассмотрим отображение

$$
S f:=\mathrm{id}+S_{E}^{(t, s, k)}(f-\mathrm{id}), \quad \text { где } \widetilde{T}_{C}^{(t, 0)}=: \widetilde{T}_{C}^{t}, \quad T^{(t, s)}:=T^{(t, s, 0)},
$$

$\infty>t \geqslant 1$. Оператор $S$ действует на функцию $([A] T f)\left(y ; a, b, \ldots, z ; \zeta_{a}, \zeta_{b}, \ldots, \zeta_{z}\right)$ таким образом, что выполняется следуюшее равенство: $S([A] T f)(y ; a, \ldots, z ; 0, \ldots, 0)=$ $\operatorname{SATf}(y)$, где $k=\operatorname{deg}(A), \quad A=A_{n}$.

Введем обозначения $F:=S[A] T$ и $\widetilde{F}:=S[A] \widetilde{T}$ на $W_{\text {id }}$.

2.6. Лемма. Существует открыто-замкнутая подгруппа $W$ в $\operatorname{Dif}(t, M \mid h)$ или в $G(t, M)$ и открыто-замкнутая окрестность $V$ нуля в $E\left(t,-2 s n, M^{2 s n+1} \rightarrow \mathbf{K}^{n}\right)$, для которых $\widehat{F}: W \rightarrow V$ - равномерныц изоморфизм, где $\widehat{F}=F$ или $\widehat{F}=\widetilde{F}$, соответственно, $c \infty>t \geqslant 1$ (см. $n .2 .3$ и 2.5).

ДокаЗАТЕЛЬСтво. $W(\tau):=\left(f \in W_{\mathrm{id}}: \rho_{0}^{\tau}(f, \mathrm{id}) \leqslant 1 / p\right)$ - открыто-замкнутая подгруппа в $\operatorname{Dif}(\tau, M \mid h)$, где $\tau=t$ или $\tau=\operatorname{an}_{r}$. Тогда

$$
[A] \in C\left(1, W\left(\operatorname{an}_{r}\right) \rightarrow E\left(\operatorname{an}_{r},-2 s n, M^{2 s n+1} \rightarrow \mathbf{K}^{n}\right)\right) \text { и }[A]^{\prime}(\mathrm{id})=[\Delta]^{s n} .
$$

2.7. Пусть $f(x) \in C((t, s-1), M \rightarrow \mathbf{K}), n \in \mathbf{N}, \quad M$ открыто-замкнуто в шаре $\mathbf{B}\left(\mathbf{K}^{n}, 0, r\right), 0<r<\infty, l=[t], b=\{t\}$, антидифференцирование $P(l, s)$ задается формулой

$$
P(l, s) f(x):=\sum_{\left(m \in \mathbf{N}_{0}^{n}, j=j^{\prime}+s^{\prime} \bar{u}, 0 \leqslant s^{\prime} \leqslant s, 0 \leqslant\left|j^{\prime}\right|<l\right)} \partial^{j} f\left(x_{m}\right)\left(x_{m+\bar{u}}-x_{m}\right)^{(j+\bar{u})} /(j+\bar{u}) !
$$

где $\partial^{j}=\partial_{1}^{j(1)} \ldots \partial_{n}^{j(n)}, j=(j(1), \ldots, j(n)), \bar{u}=(1, \ldots, 1) \in \mathbf{N}^{n}, x_{m}=\sigma_{m}(x)$, a $\left\{\sigma_{m}\right.$ : $\left.m \in \mathbf{N}_{0}^{n}\right\}$ - приближение единишы в $M[10]$. 
2.8. Tеорема. Ecлu $f \in C((t, s-1), M \rightarrow \mathbf{K}), l \in \mathbf{N}, 1 \leqslant t=l+b, \quad\{t\}=b \in \mathbf{Q}$, mo

$$
\begin{aligned}
P(l, s) f(x)-P(l, s) f(y)= & \sum_{\left(j=j^{\prime}+s^{\prime} \bar{u}, 0 \leqslant\left|j^{\prime}\right| \leqslant l, 1 \leqslant s^{\prime} \leqslant s\right)}\left(\partial^{j-\bar{u}} f(y)\right)\left[\frac{(x-y)^{j}}{j !}\right]+ \\
& +\sum_{\left(v=v^{\prime}+s \bar{u},\left|v^{\prime}\right|=l\right)}(x-y)^{v} R(n, v ; x, y),
\end{aligned}
$$

где $R(n, v ; x, y)$ и $R(n, v ; x, y) / j_{b}(\zeta)$ ( в последнем случае $x-y=\zeta e_{i}, \quad \zeta \in \mathbf{K}$ для любых $i=1, \ldots, n)$ являются непрерывными функциями, равными нулю на диагонали.

ДокаЗАТЕЛЬство. Пусть $l \in \mathbf{N}, f \in C((t, s-1), M \rightarrow \mathbf{K})$ и $\partial^{\bar{u}} f(x) \in C((t, s-1)$, $M \rightarrow \mathbf{K})$, а также

$$
\begin{aligned}
f(x)= & f(y)+\sum_{\left(1 \leqslant\left|j^{\prime}\right| \leqslant l, j=j^{\prime}+s^{\prime} \bar{u}, s^{\prime} \in\{0,1, \ldots, s\}\right)} \frac{\partial^{j} f(y)}{j !}(x-y)^{j}+ \\
& +\sum_{\left(\left|j^{\prime}\right|=l, j=j^{\prime}+s \bar{u}\right)}(x-y)^{j} R(n, j ; x, y),
\end{aligned}
$$

где $R(n, j ; x, y) \in C(b, M \times M \rightarrow \mathbf{K})$ - функция, равная нулю на диагонали $((x, y) \in$ $\left.M^{2}: x=y\right)$. Тогда для любых $z \in M($ см. $\S 78.3$ и $\S 78$. А в [10]) имеем

$$
\lim _{x, y \rightarrow z} \bar{J}_{j}^{l, q} f\left(x, y ; \zeta_{1}, \ldots, \zeta_{q n}\right) \frac{(x-y)^{l-1}}{j_{b}(\zeta)}=\frac{\partial^{q \bar{u}}\left(\bar{\Phi}^{t} f\right)(z, \ldots, z)}{(q n) !},
$$

где

$$
\begin{aligned}
\left(\bar{J}_{j}^{k, q} f\right. & \left.\left(x, y ; \zeta_{1}, \ldots, \zeta_{q n}\right)\right)(x-y)^{k}:= \\
& :=\left(\bar{\Phi}^{k+q n} f\right)\left(y ; x-y, \ldots, x-y, h_{1}, \ldots, h_{q n} ; 0, \ldots, 0,1, \ldots, 1, \zeta_{1}, \ldots, \zeta_{q n}\right)
\end{aligned}
$$

с $j$ нулями в $(\ldots), j_{b}(\zeta)-$ функция вида $j_{b}(\zeta)=p^{b \times \operatorname{ord}_{p} \zeta}$ при $\zeta \neq 0$ и $j_{b}(0):=0$ для $0<b<1$, причем $|\zeta|=p^{-\operatorname{ord}_{p} \zeta}, j_{1}(\zeta):=\zeta, x=y+\zeta e_{i}$, а векторы удовлетворяют равенствам $h_{1}=\cdots=h_{q}=e_{1}, h_{q+1}=\cdots=h_{2 q}=e_{2}, h_{q(n-1)+1}=\cdots=h_{q n}=e_{n}$.

В силу формулы Тейлора имеются непрерывные функции $A(j, v ; *)$ и $A(j, v ; x, y) / j_{b}(\zeta)$ (для последних $\left.x-y=\zeta e_{i}, i=1, \ldots, n\right)$ такие, что выполняются равенства

$$
\partial^{j} f\left(x_{m}\right)=\sum_{|q|=0, \ldots, l-|j|} \partial^{j+q} f(y) \frac{\left(x_{m}-y\right)^{q}}{q !}+\sum_{|v|=l-|j|}\left(x_{m}-y\right)^{v} A\left(j, v ; x_{m}, y\right)
$$

$$
\begin{aligned}
P(l, s) f(x) & =\sum_{\left(j=j^{\prime}+s^{\prime} \bar{u}, 0 \leqslant s^{\prime}<s\right)} \frac{\partial^{j} f(y)}{(j+\bar{u}) !}\left[(x-y)^{j+\bar{u}}+(-1)^{n}\left(x_{0}-y\right)^{j+\bar{u}}\right]+ \\
+ & \sum_{\left(m \in \mathbf{N}_{0}^{n}, j=j^{\prime}+s^{\prime} \bar{u}, 0 \leqslant s^{\prime}<s,|v|=l-|j|\right)}\left(x_{m}-y\right)^{v}\left(x_{m+\bar{u}}-x_{m}\right)^{j+\bar{u}} \frac{A\left(j, v ; x_{m}, y\right)}{(j+\bar{u}) !}
\end{aligned}
$$

(см. $\S 80.3$ и $\S 81.2$ в [10]).

2 Теоретическая и математическая физика, т. 119, № 3, 1999 г. 
2.9. Теорема. Для $1 \leqslant t<\infty$ каждая $f \in C((t, s-1), M \rightarrow \mathbf{K})$ uмеeт $C((t, s)$, $M \rightarrow \mathbf{K})$-антипроизводную такую, что $\partial^{\bar{u}}(P(l, s) f)(x)=f(x)$ для любьх $x \in M$, и если $x^{i}=x_{0}^{i}=0$ для некоторого $i$, то $\partial^{j}(P(l, s) f)(x)=0$ для любих $j=(j(1), \ldots, j(n))$ с $j(k)<2$ при всех $k=1, \ldots, n$.

ДокАЗАТЕЛЬСТво. Пусть $b \in \mathbf{Q}$, тогда в соответствии с теоремой п. 2.8 имеем

$$
P(l, s) f(x)-P(l, s) f(y)=\sum_{\left(\left|j^{\prime}\right|=0, \ldots, l ; j=j^{\prime}+s^{\prime} \bar{u}, 0 \leqslant s^{\prime}<s\right)} \partial^{j} f(y) \frac{(x-y)^{j+\bar{u}}}{(j+\bar{u}) !}+S,
$$

где

$$
S:=\sum_{v=v^{\prime}+s \bar{u},\left|v^{\prime}\right|=l} R(l, v ; x, y)(x-y)^{v},\left.\quad\left\{\frac{\partial^{n} S}{\partial x^{1} \ldots \partial x^{n}}\right\}\right|_{\{x=y\}}=0
$$

(в соответствии с $\S 29.12$ из [10]). Для $t \in \mathbf{R} \backslash \mathbf{Q}$ воспользуемся нормой

$$
\|F\|_{C(t, M \rightarrow \mathbf{K})}=\sup _{(0 \leqslant \omega \leqslant t, \omega \in \mathbf{Q})}\|F\|_{C(\omega, M \rightarrow \mathbf{K})} .
$$

2.10. Пусть $f \in C_{0}\left(t^{\prime}, M \rightarrow \mathbf{K}\right)=: Z, F$ - непрерывный $\mathbf{K}$-линейный функционал на $C_{0}(t, M \rightarrow \mathbf{K})$ с $l$ и $l^{\prime} \in \mathbf{N}, 1>b \geqslant 0, b \in \mathbf{Q}, 1>c \geqslant 0, t^{\prime}=l^{\prime}+c=2 t, t=l+b$. Тогда

$$
\left\langle\left(\bar{\Phi}^{v} F\right) \mid f\right\rangle:=(-1)^{s^{\prime}}\left\langle F_{v} \mid\left(\bar{\Phi}^{v} f\right)\right\rangle,
$$

где

$$
\left\langle F_{v}\right|\left(\bar{\Phi}^{v} f\right)\left(x ; h_{1}, \ldots, h_{v} ; 0, \ldots, 0\right\rangle=\left\langle F \mid\left(\bar{\Phi}^{v} f\right)\left(x ; h_{1}, \ldots, h_{v} ; 0, \ldots, 0\right)\right\rangle ;
$$

$v \leqslant t$ и $s^{\prime}=[v]+\operatorname{sign}\{v\}$. При $\operatorname{dim}_{\mathbf{K}} M=n \in \mathbf{N}$ и $s \in \mathbf{N}_{0}$ определим $C_{0}((-t, s), M \rightarrow \mathbf{K})$ как пространство $F \in C_{0}\left(t^{\prime}, M \rightarrow \mathbf{K}\right)^{*}$ с непрерывными линейными функционалами $\left(\bar{\Phi}^{v} F\right)\left(x ; h_{1}, \ldots, h_{v} ; \zeta_{1}, \ldots, \zeta_{v}\right)$ на $C_{0}\left(\left(t^{\prime}, s-s^{\prime \prime}\right), M^{v+1} \rightarrow \mathbf{K}\right)$ для любых $v=n s^{\prime \prime}$ с $s^{\prime \prime} \in$ $(1,2, \ldots, s)$, для которых

$$
\lim _{k \rightarrow \infty} \sup _{\left(x, x+h_{1} \zeta_{1}+\cdots+h_{i} \zeta_{i} \in M ; i=1, \ldots, v\right)}\left(\left|\left\langle F_{v} \mid\left(\bar{\Phi}^{v} f_{k}\right)\left(x ; h_{1}, \ldots, h_{v} ; \zeta_{1}, \ldots, \zeta_{v}\right)\right\rangle\right|\right)=0,
$$

где $h_{1}=\cdots=h_{s^{\prime \prime}}=e_{1}, \ldots, h_{(n-1) s^{\prime \prime}+1}=\cdots=h_{n s^{\prime \prime}}=e_{n}$ при $0<s^{\prime \prime} \leqslant s$.

БП $C_{0}((-t, s), M \rightarrow \mathbf{K})$ является сепарабельным с нормой

$$
\|F\|=\sup _{(|m|=0,1, \ldots ; j \in \Lambda)}\left|F\left(\left.\bar{Q}_{m}\right|_{U_{j}}\right)\right| \frac{J_{j}((0, s), m)}{J_{j}(t, m) \min \left(1, r_{j}\right)} .
$$

2.11. Для $0<b \in \mathbf{R} \backslash \mathbf{Q}, l \in \mathbf{N}_{0}, t=l+b$ пусть $C_{0}((-t, s), M \rightarrow \mathbf{K})$ является пополнением $C_{0}((-[t]-1, s), M \rightarrow \mathbf{K})$ по норме

$$
\|F\|_{C_{0}((-t, s), M \rightarrow \mathbf{K})}:=\sup _{t<\omega \in \mathbf{Q}, \omega<[t]+1}\|F\|_{C_{0}((-\omega, s), M \rightarrow \mathbf{K})} .
$$


Для подмногообразия $\widetilde{M}$ в $M$ (см. п. 2.3 и 2.10 ) сушествует подмногообразие $\bar{M}_{0}, \widetilde{M} \subset$ $\bar{M}_{0} \subset M$, причем $\bar{M}_{0}$ открыто-замкнуто в $M$ и $\operatorname{At}(M)$ индуцирует $\operatorname{At}\left(\bar{M}_{0}\right)$, т. е.

$$
\bar{\phi}_{0, j}\left(\bar{U}_{0, j}\right)=\tilde{\phi}_{j}\left(\widetilde{U}_{j}\right) \times \mathbf{B}\left(\mathbf{K}, y_{j}, \tilde{r}_{j}\right) \subset \phi_{l}\left(U_{l}\right)=\mathbf{B}\left(X, x_{l}, r_{l}\right),
$$

где $\left(\bar{U}_{0, j}, \bar{\phi}_{0, j}\right)$ и $\left(\widetilde{U}_{0, j}, \tilde{\phi}_{0, j}\right)$ - карты атласов $\operatorname{At}\left(\bar{M}_{0}\right)$ и $\operatorname{At}(\widetilde{M}), U_{l} \supset \bar{U}_{0, j} \supset \widetilde{U}_{j}$. Поэтому для любой функции $f \in C_{0}(t, \widetilde{M} \rightarrow \mathbf{K})$ сушествует функция $\tilde{f} \in C_{0}(t, M \rightarrow \mathbf{K})$ с ограничениями $\left.\tilde{f}\right|_{\bar{M}}=\left.f\right|_{\bar{M}}$ и $\left.\tilde{f}\right|_{U_{j}}$, не зависящими от нормальной координаты, соответствуюшей множителю $\mathbf{B}\left(\mathbf{K}, y_{j}, \tilde{r}_{j}\right)$ для любого $j$, такая, что $\left.\tilde{f}\right|_{\left(M \backslash \bar{M}_{0}\right)}=0$. Тогда $\left.(A F)\right|_{\bar{M}}=0$, если $\langle(A F) \mid \tilde{f}\rangle=0$ для любой пары таких функций $f$ и $\tilde{f}$, где $A$ - дифференциальный оператор. Определим $C_{0}((-t, s), M \rightarrow N)$ и замкнутые подгруппы $\operatorname{Dif}(\tau, M \mid h)$ в $G(\tau, M)$ для $2 v(h, n)>t$ аналогично п. 2.2 и 2.3 .

\section{3. МЕРЫ И ПРЕДСТАВЛЕНИЯ}

3.1.1. Пусть $G$ обозначает хаусдорфову группу, а мера $\mu: A f(G, \mu) \rightarrow \mathbf{Y}$ определена на пополнении $A f(G, \mu)$ борелевской $\sigma$-алгебры $B f(G)$ по $\mu[4,11]$, где $\mathbf{K}_{q}$ - локальное поле, $\mathbf{K}_{q} \supset \mathbf{Q}_{q} c q \neq p, Y=\mathbf{R}$ или $Y=\mathbf{K}_{q}$. Рассмотрим преднорму

$$
\|f\|_{\psi}:=\sup _{x \in G}(|f(x)| \psi(x))
$$

и зададим функцию

$$
N_{\mu}(x):=\inf \left(\|U\|_{\mu}: U \in B_{\mathrm{co}}(G), x \in U\right)
$$

для соответствуюших функций $f: G \rightarrow \mathbf{K}_{q}, \psi: G \rightarrow[0, \infty)$ и для $\mathbf{K}_{q}$-значной меры, где

$$
\|A\|_{\mu}:=\sup \left(|\mu(B)|_{\mathbf{K}_{q}}: B \subset A, B \in A f(G, \mu)\right)
$$

для любого элемента $A$-алгебры $A f(G, \mu)$, а $B_{\text {со }}(G)$ - алгебра открыто-замкнутых подмножеств в $G,\|\mu\|:=\|G\|_{\mu}$. Мера $\nu: A f(G, \mu) \rightarrow \mathbf{K}_{q}$ абсолютно непрерывна относительно $\mu: \nu \ll \mu$, если сушествует функция

$$
f \in L(\mu):=L\left(G, \mu, \mathbf{K}_{q}\right)
$$

такая, что

$$
\nu(B)=\int_{B} f(x) \mu(d x)
$$

для любых $B \in B_{\text {со }}(G)$, где $L(\mu)-$ БП измеримых функций $f: G \rightarrow \mathbf{K}_{q}$, для которых $\|f\|_{L(\mu)}:=\|f\|_{N_{\mu}}<\infty$. Если $\mu \ll \nu$ и $\nu \ll \mu$, то они эквивалентны: $\mu \sim \nu$ (в случае $Y=\mathbf{R}$ см. [11]). Мера $\mu$ называется левоквазиинвариантной относительно плотной подгруппы $G^{\prime}$ в $G$, если $\mu_{\phi}(*)$ эквивалентна $\mu(*)$ для любых $\phi \in G^{\prime}$, где $\mu_{\phi}(E):=\mu\left(\phi^{-1} E\right)$ для любых $E \in A f(G, \mu), \rho_{\mu}(\phi, g):=\mu_{\phi}(d g) / \mu(d g)$ - фактор квазиинвариантности. 
3.1.2. Функция $f: \mathbf{B}(\mathbf{K}, 0,1) \rightarrow Y$ назьвается псевдодифференцируемой порядка $b$, если существует интеграл

$$
P D_{c}(b, f(x)):=\int_{\mathbf{B}(\mathbf{K}, 0,1)}[(f(x)-f(y)) \times g(x, y, b)] d v(y),
$$

где $g(x, y, b):=|x-y|^{-1-b}$ для $Y=\mathbf{R}$ и $g(x, y, b):=q^{(-1-b) \times \operatorname{ord}_{p}(x-y)}$ для $Y=\mathbf{K}_{q}$ с соответствуюшей мерой Хаара $v$ на $\mathbf{K}$ со значениями в $Y, b \in \mathbf{C}$ (см. [10] и $\S 2.1$ из [5], для $Y=\mathbf{R}$ см. [3, 12]).

3.1.3. Рассмотрим $C^{\tau}$-отображение

$$
\mathrm{S}: \mathbf{B}(\mathbf{K}, 0,1) \times G \rightarrow G
$$

такое, что

$\mathrm{S}\left(\mathbf{B}(\mathbf{K}, 0,1) \times G^{\prime \prime}\right) \subset G^{\prime \prime}, \frac{\partial \mathrm{S}(\zeta, f)}{\partial \zeta} \in T G^{\prime \prime} \quad$ и $\left.\frac{\partial \mathrm{S}(\zeta, f)}{\partial \zeta}\right|_{\zeta=0}:=A_{f} \in T_{f} G^{\prime \prime}, \quad \mathrm{S}(0, f)=f$.

Введем обозначение: $\widetilde{\mathrm{S}}(\zeta, \mu)(B):=\mu(\mathrm{S}(-\zeta, B))$ для любых $B \in B f(G)$, где $G^{\prime \prime}$ - плотная подгруппа в $G^{\prime}$. Мера $\mu$ называется псевдодифференцируемой порядка $b$ относительно $\mathrm{S}$, если существует интеграл $P D_{c}(b, \tilde{\mathrm{S}}(\zeta, \mu)(B))$ по $\zeta \in \mathbf{B}(\mathbf{K}, 0,1)$ для любых $B \in B f(G)$, где $1 \leqslant \tau$ или $\tau=\operatorname{an} \bar{R}, \infty>\bar{R}>0$.

3.2. ЛЕмма. Пусть $f \in G((t, s-1), M), \quad M=\mathbf{B}\left(\mathbf{K}^{n}, 0,1\right) u \rho^{(t, s-1)}(f, \mathrm{id}) \leqslant c c$ постоянной

$$
c:=\min _{\left(\left|j^{\prime}\right|=0, \ldots, l ; j=j^{\prime}+s^{\prime} \bar{u} ; s^{\prime} \in\{0,1, \ldots, s-1\}\right)} \frac{1}{p}\left|\prod_{i=1}^{n}(j(i)+1)\right| .
$$

Тогда

$$
Q((l, s) ; f):=P(l, s)(f-\mathrm{id})+\mathrm{id}=: g \in G((t, s), M)
$$

$u \rho^{(t, s)}(g, \mathrm{id}) \leqslant 1 / p$, əде $l=[t]$.

ДОКАЗАТЕЛЬСТво. Пусть $h=f-\mathrm{id}$, тогда в соответствии с п. 2.8 выполняется неравенство: $\|P(l, s) h\|_{C((t, s), M)} \leqslant c$, т.к. $\left|\partial^{j-\bar{u}} h(x) / j !\right| \leqslant c$ для любых $j=j^{\prime}+s^{\prime} \bar{u}$, следовательно, $\rho^{(t, s)}(g, \mathrm{id}) \leqslant 1 / p$, поэтому $g \in G((t, s), M)$.

3.3. ЛЕммА. Пусть $0 \leqslant l<2 s n+1,0 \leqslant b<1$,

$$
k=z+l \geqslant 1, \quad z \in \mathbf{N}_{0}, \quad R(f):=\partial^{z \bar{u}} \widehat{F} \widetilde{Q}(f),
$$

әде отображение

$$
\widetilde{Q}(f):=Q((l, z) ; Q((l, z-1) ; \ldots ; Q((l, 1) ; f) \ldots))
$$

действует по переменной у подобно оператору $S$ из $n .2 .5, t=l+b$ (cм. $n .2 .5,2.6 u$ 2.10). Тогда для $\widehat{F}=F($ или $\widehat{F}=\widetilde{F})$ отображсение $R$ является диффеоморфизмом открьто-замкнутой подгруппь $W$ из $G:=\operatorname{Dif}(t, M \mid h)($ или $G:=G(t, M)$, соответственно) на открыто-замкнутую окрестность $V$ нуля в $E\left(t,-2 s n, M^{2 s n+1} \rightarrow\right.$ $\left.\mathbf{K}^{n}\right)$. 
ДокАЗАТЕЛЬСТво. В силу леммы 3.2 отображение $\widetilde{Q}(f)$ является изометрией БМ $M$ и принадлежит $\operatorname{Dif}((t, z), M \mid h)$ с $\rho^{(t, z)}(\widetilde{Q}(f), \mathrm{id}) \leqslant 1 / p$, когда

$$
\rho^{t}(f, \mathrm{id}) \leqslant \min _{\left(\left|j^{\prime}\right|=0, \ldots, l ; j=j^{\prime}+s^{\prime} \bar{u} ; 0 \leqslant s^{\prime} \leqslant s\right)} \frac{1}{p}\left[\prod_{i=1}^{n}|(j(i)+1) \ldots(j(i)+z)|\right]=: c .
$$

Поэтому, $R^{\prime}(\mathrm{id}) h=\partial^{z \bar{u}} S[\Delta]^{s n} T P h$, т.к. $[A]^{\prime}=[\Delta]^{s n}$ и $[\Delta]^{s n} \mathrm{id}=0$, а также $Q((l, z) ; f)=\mathrm{id}+P(l, z) h$ для $f=\mathrm{id}+h \in W$ с производной $Q^{\prime}((l, z) ; \mathrm{id}) h=P(l, z) h$, причем $\widetilde{Q}^{\prime}(\mathrm{id}) h=P h$ для отображения $\widetilde{Q}(f)=\mathrm{id}+P h$, а оператор $R^{\prime}(\mathrm{id})$ обратим, где $P:=P(l, z) \ldots P(l, 1)$ и $W:=\mathbf{B}(G, \mathrm{id}, c)$.

3.4. Каждая $f \in C_{0}(t, M \rightarrow X)$ имеет разложение

$$
\left.f(x)\right|_{U_{j}}=\left.\sum_{\left(i \in \mathbf{N}, n \in \mathbf{N}_{0}\right)} f^{i}(n ; x)\right|_{U_{j}} e_{i} \tilde{z}(n),
$$

где $\left\{e_{i} \tilde{z}(n)\left(\left.\bar{Q}_{m}(x)\right|_{U_{j}}\right): i, n, \operatorname{Ord}(m)=n, j\right\}$ - ортогональный базис, более того,

$$
\left.f_{n}(x)\right|_{U_{j}}:=\left.\sum_{i} f^{i}(n ; x)\right|_{U_{j}} e_{i} \in C_{0}\left(t, U_{j} \rightarrow X\right),
$$

где

$$
X_{\tilde{z}(n)}:=\left\{f_{n}(x):\left.f_{n}\right|_{U_{j}} \in C_{0}\left(t, U_{j} \rightarrow X\right)\right\}
$$

- это БП с нормой, индуцированной из $C_{0}(t, M \rightarrow X)$, причем

$$
\left.f^{i}(n ; x)\right|_{U_{j}}:=\left.\sum_{\left(\operatorname{Ord} m=n, m=(m(1), \ldots, m(n)), m(j) \in \mathbf{N}_{0}\right)} a\left(m,\left.f^{i}\right|_{U_{j}}\right) \bar{Q}_{m}(x)\right|_{U_{j}},
$$

a $X$ - это БП из п. 2.2 ; полагаем $\left.\bar{Q}_{m}(x)\right|_{U_{j}}=0$ при $x \in M \backslash U_{j}$.

3.5.1. Для $M$ зафиксируем последовательность $\left\{M_{n}: n \in \mathbf{N}_{\mathbf{0}}\right\}$ подмногообразий в $M$ таких, что $M_{n} \hookrightarrow M_{n+1} \hookrightarrow \cdots \hookrightarrow M$ для любых $n, \operatorname{dim}_{\mathbf{K}} M_{n}=\beta(n) \in \mathbf{N}$ для любых $n \in \mathbf{N}_{0}, \bigcup_{n} M_{n}$ плотно в $M$, где $\beta(n)<\beta(n+1)$ для любых $n$ и сушествует $n_{0} \in \mathbf{N}$ с $\beta(n)=n$ для любых $n>n_{0}$. Пусть имеется последовательность $\{s(n) \in \mathbf{N}: n \in \mathbf{N}\}$, $s(n) \leqslant s(n+1)$ для любых $n$. Определим оператор

$$
A(f):=\sum_{\left(n \in \mathbf{N}, k=(k(1), \ldots, k(\beta(n))) ; k(j) \in \mathbf{N}_{0}, n \in \mathbf{N}\right)} q(k) A\left(k ; f^{\prime}, \bar{P}(\beta(n)) f\right) \tilde{z}(\beta(n)),
$$

где $|(k(1), \ldots, k(\beta(n)))|=s(n) \beta(n)$, причем

$$
\sum_{(\operatorname{Ord}(k) \leqslant n,|k|=s(n) \beta(n))} q(k)=: \tilde{t}(n),
$$

а также $0<c \leqslant|\tilde{t}(n)| \leqslant c^{\prime}<\infty$ для любых $n$ с фиксированными $c$ и $c^{\prime}, \bar{P}(n)-$ проекторы на $X_{\tilde{z}(n)}$, т.е. $\bar{P}(n) f(x)=f_{n}(x)$ (см. п. 2.5.3 и 3.4 с $X=Y$ ). 
3.5.2. Определим отображение

$$
R(f):=\sum_{\left(n \in \mathbf{N}_{\mathbf{0}}, k=(k(1), \ldots, k(\beta(n))), k(j) \in \mathbf{N}_{0}\right)} q(k) R(k, n ; f) \tilde{z}(\beta(n)),
$$

где

$$
R(k, n ; f):=\partial^{z \bar{u}} F(k, n ; \widetilde{Q}(f ; n)), \quad F(k, n ; f):=S[A]\left(k ; g^{\prime}, \bar{P}(n) g\right)
$$

с $g=\widehat{T} f$ и $l+z \geqslant 1$ (см. п. 3.3), а оператор $Q((l, s) ; *)$ действует на функцию $f_{\beta(n)}(x)$ по переменным $\left(x^{1}, \ldots, x^{\beta(n)}\right)$, при этом

$$
\widetilde{Q}(f ; n):=Q\left((l, z) ; Q\left((l, z-1) ; \ldots ; Q\left((l, 1) ; f_{\beta(n)}(x)\right) \ldots\right)\right) .
$$

Операторы $\widehat{T}$ и $S$ даются формулами

$$
\widehat{T} f:=\sum_{n=0}^{\infty}\left(\widehat{T}_{n} f_{n}\right) \tilde{z}_{n}, \quad S g:=\sum_{n=0}^{\infty}\left(S_{n} g_{n}\right) \tilde{z}_{n}
$$

причем $S_{n}$ и $\widehat{T}_{n}$ те же, что и операторы $S$ и $T$ для подмногообразия $M_{n}$ вместо всего многообразия $M$ в п. 2.5, а также принято соглашение: $\widehat{T}=T$, если $\widehat{T}_{n}=T_{n}$ для любых $n \in \mathbf{N}_{\mathbf{0}} ; \widehat{T}=\widetilde{T}$, если $\widehat{T}_{n}=\widetilde{T}_{n}$ для любых $n \in \mathbf{N}_{\mathbf{0}}$.

3.5.3. Пусть имеется последовательность БП $H(n)=E\left((t, z),-a, M_{n}^{a+1} \rightarrow X\right)$ с $a=$ $2 s(n) \beta(n)$ для любых $n \in \mathbf{N}_{\mathbf{0}}$. Тогда БП $c_{0}(H(n): n)$ (см. п. 2.2.1) обозначим через $E(t$, inf $)$. Зададим подгруппу

$$
\begin{aligned}
G(t, M \mid h):= & \left\{f: f \in G(t, M),\left\{\left(f_{i, j}^{u}(x)-\operatorname{id}_{i, j}^{u}(x)\right): u=1, \ldots, \beta(n)\right\} \in\right. \\
& \left.\in C_{0}\left(t, U_{j} \rightarrow X \mid h(n)\right), n \in \mathbf{N}_{0}\right\}
\end{aligned}
$$

для последовательности $h:=\left\{h(n): h(n)=[s(n) \beta(n) / 2], n \in \mathbf{N}_{0}\right\}$ с помошью подпространств

$$
\begin{array}{r}
C_{0}\left(t, U_{j} \rightarrow X \mid h(n)\right):=\left\{f \in C_{0}\left(t, U_{j} \rightarrow X\right)\left|\Delta_{n}^{l} f^{u}\right|_{\widetilde{M}_{n}}=0\right. \\
\text { для любых } l=0, \ldots, v(h(n), n)\},
\end{array}
$$

где $s(n)=\max \left(2,\left[\bar{c}^{\prime} n\right]\right), 0<\bar{c}^{\prime}=$ const, $\bar{c}^{\prime} \in \mathbf{R}, \widetilde{M}_{n}:=\widetilde{M} \cap M_{n}$ (см. п. 2.3).

3.6. ТеоремА. Групnы $G_{1}:=G(t, M)$ u $G_{2}:=G(t, M \mid h)$ из $n$. 3.5 для любих $0 \leqslant t \in \mathbf{R}$ или $t=\operatorname{an}_{r}$ и $b^{\prime} \in \mathbf{C}$ имеют квазиинвариантние и псевдодифферениируемые порядка $b^{\prime}$ меры $\mu$ со значениями в $[0, \infty)$ и $\mathbf{Q}_{q}($ для простых чисел $q \neq p)$ относительно плотных подгрупп $G_{\eta}^{\prime}$ для $\eta=1,2$. Если $M$ компактно, то $G_{\eta}^{\prime} \supset$ $G\left(t^{\prime}, M\right) \cap G_{\eta}$ nрu $t^{\prime}=t+s \quad \partial \Omega_{g} 0 \leqslant t \in \mathbf{R}, s \geqslant 2, u G_{\eta}^{\prime} \supset G\left(\operatorname{an}_{R}, M\right) \cap G_{\eta}$ nрu $t=\operatorname{an}_{r}$ $c R>r>0$. 
ДокАЗАтЕльСтво. Сушествует открыто-замкнутая подгруппа $W$ в $G_{\eta}$ такая, что $W$ дифффеоморфна открыто-замкнутой окрестности $V$ нуля в БП $Z$, при этом $R(W)=V$. $R$ задано в п. 3.5.2 с помошью оператора для $\widehat{T}=T$ (или $\widehat{T}=\widetilde{T}$ ), где $0 \leqslant t \in \mathbf{R}$ или $t=$ $\operatorname{an}_{r}$ (при $M \subset \mathbf{B}(X, 0, r)$ в последнем случае). Имеется также вложение $Z \hookrightarrow E(t, \mathrm{inf})$, т.к. в соответствии с п. $2.6 ; 2.11 ; 3.3$ и 3.5 для любого $n>n_{0}$ операторы

$$
\begin{aligned}
\tilde{t}(n) \partial^{z \bar{u}(n)} S\left[\Delta_{n}\right]^{s(n) n} T \widetilde{Q}(* ; n): C_{0}\left(t, M_{n} \rightarrow X \mid h(n)\right) \rightarrow \\
\quad \rightarrow E\left((t, z),-2 s(n) n, M_{n}^{2 s(n) n+1} \rightarrow X\right) \text { и } \tilde{t}(n)\left[\Delta_{n}\right]^{s(n) n}
\end{aligned}
$$

кратны изометричным операторам с постоянными множителями. Возьмем подгруппу

$$
\begin{gathered}
G_{\eta}^{\prime}:=\left\{f \in G_{\eta}:\left(f^{i}(n ; x)-\mathrm{id}^{i}(n ; x)\right)=: g^{i}(n ; x) \in C_{0}\left(t_{n}, M_{n} \rightarrow \mathbf{K}\right)\right. \text { и } \\
\left.\left|a\left(m ;\left.g^{i}(n ; x)\right|_{U_{j}}\right)\right| J_{j}\left(t_{n}, m\right) \leqslant c(f) p^{v^{\prime}(m, j, i)}\right\},
\end{gathered}
$$

где $c(f)>0$ - постоянные, $v^{\prime}(m, j, i)=-c^{\prime} i-c^{\prime} n-c^{\prime \prime} j, n=\operatorname{Ord}(m), c^{\prime}=$ const $>0$ и $c^{\prime \prime}=\mathrm{const} \geqslant 0\left(c^{\prime \prime}>0\right.$ при $\left.\Lambda=\omega_{0}\right), t_{n}=t+s(n)$ при $0 \leqslant t<\infty$; а в аналитическом случае $t=\operatorname{an}_{r}$ выполняются условия $t_{n}=\operatorname{an}_{R_{n}}$ и $R_{n}>r \mathrm{c} \underline{\lim }_{n \rightarrow \infty}\left(R_{n} / n\right)>0, c^{\prime \prime}=0$ и $j=1$.

Тогда имеется ультраметрика на $G_{\eta}^{\prime}$

$$
d_{\eta}(f, \mathrm{id})=\sup _{m, n, j}\left\{\left|a\left(m ;\left.g^{i}(n ; x)\right|_{U_{j}}\right)\right| J_{j}\left(t_{n}, m\right) p^{-v^{\prime}(m, j, i)}\right\}
$$

(см. также $\S$ I.2.16, I.3.7 и II.2.2 из [8]). Для построения $\mu$ на $G$ достаточно ее построить на $W$, т.к. существуют счетное семейство $\left\{g_{i}: i=1,2, \ldots\right\} \subset G^{\prime}$ и дизъюнктные открыто-замкнутые покрытия

$$
\left\{V_{j}: V_{j}:=g_{j} W_{j}, W_{j} \subset W \text { при } j>1 \text { и } V_{1}=W\right\}
$$

группы $G_{\eta}$ и

$$
\left\{V^{\prime}{ }_{j}: V^{\prime}{ }_{j}:=g_{j} W^{\prime}{ }_{j}, W^{\prime}{ }_{j} \subset W^{\prime} \text { при } j>1 \text { и } V^{\prime}{ }_{1}=W^{\prime}\right\}
$$

группы $G_{\eta}^{\prime}$, где $W^{\prime}$ открыто-замкнуто в $G_{\eta}^{\prime}$ и $W^{\prime} \subset G_{\eta}^{\prime} \cap W$. Поэтому мера

$$
\mu(H):=\sum_{j \in \mathbf{N}} y\left(g_{j}^{-1}\left(H \cap V_{j}\right)\right) x^{j},
$$

будет квазиинвариантной и псевдодифференшируемой относительно $G_{\eta}^{\prime}$, если $y$ квазиинвариантна и псевдодифференшируема относительно $G_{\eta}^{\prime} \cap W ; 0<x<1$ для поля $\mathbf{R}$ и $x=q^{n}$ с $n \in \mathbf{N}$ для $\mathbf{Q}_{q}$.

А. Для локально-компактного $M$ БП $E\left((t, z),-2 s n, M^{2 s n+1} \rightarrow \mathbf{K}^{n}\right)=: Z$ имеет базис

$$
\left\{\left.[\Delta]^{s n} \bar{Q}_{m}(x)\right|_{U_{j}} e_{i}: i=1, \ldots, n ; m \in \mathbf{N}_{\mathbf{o}}^{l}, l=\operatorname{Ord}(m) \leqslant \operatorname{dim}_{\mathbf{K}} M=: n ; j \in \Lambda\right\},
$$


что индуцирует изоморфизм БП $Z$ с $c_{0}\left(\omega_{0}, \mathbf{K}\right)$. На $Z$ сушествуют меры $\nu$ вида

$$
\begin{gathered}
\nu(d x)=\bigotimes_{j} \nu_{\kappa(j)}\left(d x^{j}\right), \quad x=\left(x^{j}: x^{j} \in \mathbf{K}, j \in \mathbf{N}\right) \in c_{0}\left(\omega_{0}, \mathbf{K}\right), \\
\nu_{j}\left(d x^{j}\right)=f_{j}\left(x^{j}\right) w\left(d x^{j}\right), \quad f_{j} \in L^{1}(\mathbf{K}, w, \mathbf{R}) \text { или } f_{j} \in L\left(\mathbf{K}, w, \mathbf{K}_{q}\right),
\end{gathered}
$$

$\nu_{j}(\mathbf{K})=1$ для любого $j, w$ - мера Хаара на $\mathbf{K}$ со значениями в $\mathbf{R}$ или $\mathbf{K}_{q}$, так что $w(\mathbf{B}(\mathbf{K}, 0,1))=1$. Можно взять, например,

$$
\left.f_{j}\left(d x^{j}\right)\right|_{\bar{S}(j, n)}=a(j, n), \quad \text { где } \bar{S}(j, n):=\mathbf{B}\left(\mathbf{K}, 0, p^{-j}\right) \backslash \mathbf{B}\left(\mathbf{K}, 0, p^{-j-1}\right)
$$

для $j \in \mathbf{Z}$ с $j<n$,

$$
\bar{S}(n, n):=\mathbf{B}\left(\mathbf{K}, 0, p^{-n}\right), \quad a(j, n):=2^{n(j-n-1)}\left(1-2^{-n}\right)\left(1-\frac{1}{p}\right) p^{-n}
$$

для $j<n$ и $a(n, n):=\left(1-2^{-2 n}\right) p^{-n}$ для действительной меры;

$$
a(j, n):=(1-q)\left(1-\frac{1}{p}\right) q^{2 n-1-j} p^{-n}
$$

при $j<n$ и $a(n, n):=\left(1-q^{-n}\right) p^{-n}$ для $\mathbf{K}_{q}$-значной меры. Пусть

$$
\begin{aligned}
& \kappa(b) \leqslant \kappa(b+1) \text { для любого } b \in \mathbf{N}, \\
& \kappa(b) \rightarrow \infty, \\
& p^{\left(\kappa(b)-v\left(m_{b}, j_{b}, i_{b}\right)\right)} \rightarrow 0 \text { при } b \rightarrow \infty
\end{aligned}
$$

(эти пределы берутся в $\mathbf{R}), \tilde{\psi}: \mathbf{N} \rightarrow \mathbf{L}$ - биекция, $\mathbf{L}$ - множество $\{(m, j, i)\}$ индексов базиса в $Z, \tilde{\psi}(b)=:\left(m_{b}, j_{b}, i_{b}\right), v(m, j, i):=c^{\prime}|m|+c^{\prime \prime} j, c^{\prime}=$ const $>0, c^{\prime \prime}=$ const $\geqslant 0$, $c^{\prime \prime}>0$ для $\Lambda=\omega_{0}$. Для компактного $M$ можно выбрать $0<c^{\prime}<2 / n$ и $c^{\prime \prime}=0$. Для $M$, открыто-замкнутого в $\mathbf{B}(\mathbf{K}, 0,1), 0 \in M$, и для $G=\{f \in G(l, M): f(0)=0\}$ с $l \geqslant 1$ можно взять локальный диффеоморфизм $[A]$ вида

$$
([A] g)\left(x ; e_{1} ; t\right)=\log _{p}\left(\bar{\Phi}^{1} g\right)\left(x ; e_{1} ; t\right)-\log _{p}\left(\bar{\Phi}^{1} g\right)\left(x ; e_{1} ; 0\right)
$$

(см. $\S 44.1$ в [10] и $\S 2.3$ в [5]).

Для $\phi$ и $f \in W_{\text {id }} \subset C_{0}(t, M \rightarrow X)$ выполняется равенство

$$
\partial_{j}^{l}(\phi \circ f)(x)=\phi^{\prime}(f(x)) \partial_{j}^{l} f(x)+\sum_{k=1}^{s-1}\left(\begin{array}{c}
l-1 \\
k
\end{array}\right)\left\{\partial_{j}^{k} \phi^{\prime}(f(x))\right\}\left\{\partial_{j}^{l-k} f(x)\right\}+B_{j, l}(x),
$$

причем

$$
B_{j, l}(x)=\sum_{k=s}^{l}\left(\begin{array}{c}
l-1 \\
k
\end{array}\right)\left\{\partial_{j}^{k} \phi^{\prime}(f(x))\right\}\left\{\partial_{j}^{l-k} f(x)\right\}
$$


(см. п. 2.4 и 2.5.3). Для $\tilde{z} \in T \widetilde{Q}(U)$ (с $\widetilde{Q}=I$ для $z=0)$ имеем

$$
\begin{aligned}
& A\left(i ;(\tilde{t}(\tilde{z}))^{\prime}, \tilde{t}(\tilde{z})\right)-A\left(i ; \tilde{z}^{\prime}, \tilde{z}\right)= \\
& \quad=\partial_{1}^{2 i(1)} \ldots \partial_{r-1}^{2 i(r-1)} \partial_{2 r+1}^{2 i(r+1)} \partial_{n}^{2 i(n)} \sum_{l=0}^{s-1} \chi(l, 2 i(r)) \partial_{r}^{l} B_{r, 2 i(r)-l}
\end{aligned}
$$

Для данных $g$ и $v$ отображения $\tilde{t}$ и $\tilde{z}$ определены благодаря равенству $f=R^{-1}(v)$, поэтому $f \in U$, а $\tilde{z}:=T \widetilde{Q}(f)$ вместе с $\tilde{j}:=T \widetilde{Q}(g(f))$ являются диффеоморфизмами $M$ в соответствии с п. $2.5,2.6$ и 3.2 , следовательно, $\tilde{t}(x)=\tilde{j}\left(\tilde{z}^{-1}(x)\right)$ - это диффеоморфизм. Пусть $S(g, v):=R\left(g\left(R^{-1}(v)\right)\right)-v$, причем $R=\widehat{F}$ для $z=0$, а в аналитическом случае с $[A]$ вместо $R$. Предположим, что $v \in V$ и $g \in W$, где $V:=R(U), U$ и $W$ являются открыто-замкнутыми окрестностями id в $\operatorname{Dif}(t, M \mid h)$ (или $G(t, M))$ и $\operatorname{Dif}(t+s, M \mid h)$ (или $G(t+s, M)), U$ определяется в п. 2.6 и 3.3, а замыкание $W$ в $\operatorname{Dif}(t, M \mid h)$ (или $G(t, M)$, соответственно) равно $U$.

Б. Пусть теперь $\operatorname{dim}_{\mathbf{K}} M=\aleph_{0}$. Для любых $n$ и $k=(k(1), \ldots, k(n))$ рассмотрим операторы $S(k, n ; g, f):=R(k, n ; g \circ f)-R(k, n ; f)$, где $g \in G_{\eta}^{\prime}, f \in G_{\eta}$. Тогда

$$
S(k, n ; g, f) \in E\left((t, z),-a, M^{a+1} \rightarrow X\right)=: L_{n}
$$

с $a=2 s(n) \beta(n)-s(n)$ и

$$
\mid a\left(m, S\left(k, n ;\left.g\right|_{U_{j}},\left.f\right|_{U_{j}}\right)^{i} \mid J_{j}(t, m) \leqslant C p^{v^{\prime}(m, j, i)} \frac{J_{j}(t, m)}{J_{j}\left(t_{n}, m\right)},\right.
$$

где $C=$ const $>0, V^{\prime} \subset G_{\eta}^{\prime} \cap W, 0 \leqslant t \in \mathbf{R} \mathrm{c} t+z \geqslant 1$ или $t=\operatorname{an}_{r} \mathrm{c} z=0$. Пусть $Z:=$ $\operatorname{clsp}_{\mathbf{K}} R(W)$, где $Z \subset E(t$, inf $)$, и замыкание сl берется в $E(t, \mathrm{inf}) ; R: W \rightarrow V$ - диффеоморфизм, как в начале доказательства, тогда $S(k, n ; g, f)$ является $C(\infty)$-отображением из $W \times V$ в $L_{n}$. В $Z$ имеется ортогональный базис

$$
\left\{\tilde{w}(m, j, i):=\left.\left[\Delta_{\beta(n)}\right]^{s(n) \beta(n)} \bar{Q}_{m}(x)\right|_{U_{j}} e_{i} \tilde{z}(n)\right\}
$$

с $n=\operatorname{Ord}(m) \in \mathbf{N}_{0}, i \in \omega_{0}, m \in \mathbf{N}_{0}^{n}$ и $j \in \Lambda$. Пусть $\nu$ на $Z=c_{0}\left(\omega_{0}, \mathbf{K}\right)$ того же вида, что и в пункте $\mathbf{A}$, где $\kappa(b)$ удовлетворяет условиям (1)-(3) с $v(m, j, i):=c^{\prime} i+c^{\prime} \operatorname{Ord}(m)+$ $c^{\prime}|m|+c^{\prime \prime} j, \mathrm{~L}$-множество $\{(m, j, i)\}$ индексов базиса в $Z, s(n)=\max \left(2,\left[c^{\prime} n\right]\right)$ (см. также теоремы $3.23,3.28,4.2$ и 4.3 из работы [13]). Индуцируем меру $y$ на $W \subset G_{\eta}$ (см. п. 2.5, $2.6,3.3)$, так что $y(B):=\nu(R(B))$ для любых $B \in B f(W)$.

3.7. ТеОРемА. Пусть $G$ - группа диффеоморфизмов с действительной квазиинвариантной мерой $\mu$ относительно плотной подгруппь $G^{\prime}$. Тогда $\mu$ можно выбрать таким образом, чтобы регулярное представление группы $G^{\prime}$ было топологически неприводимым. 
ДоказАтЕльСтво. Если $f \in L^{1}(G, \mu, \mathbf{C})$ удовлетворяет условию $f^{h}(g)=f(g)$ $(\bmod \mu)$ для любых $h \in G^{\prime}$, то $f(x)=$ const $\quad(\bmod \mu)$ в соответствии с $\S$ II.3.1 работы [11] и леммой 2.3 из [13], где $f^{h}(g):=f(h g), g \in G$, а мера $\mu$ задана в п. 3.6. Для любого $E \subset G$ внешняя мера ограничена: $0 \leqslant \mu^{*}(E) \leqslant \mu(G)<\infty$, следовательно, существует $F \in B f(G)$, так что $F \supset E$ и $\mu(F)=\mu^{*}(E)$, т.е. $F$ может рассматриваться как минимальная (относительно значений $\mu$ ) борелевская оболочка для $E$. В соответствии c $\S$ VIII.19.5 работы [14] мера $\mu$ эргодична.

Имеется сильно непрерывное регулярное представление

$$
T_{h} f(g):=\left(\rho_{\mu}(h, g)\right)^{1 / 2} f\left(h^{-1} g\right), \text { где } f \in H, \quad h \in G^{\prime}, \quad T: G^{\prime} \rightarrow U(H),
$$

$U(H)$ - унитарная группа пространства $H:=L^{2}(G, \mu, \mathbf{C})$. В соответствии с п. 3.6 можно выбрать $\mu$, для которой существуют интегралы $P D_{c}(l, v(\mathrm{~S}(\zeta, \phi), g))$ любого порядка $l \in \mathbf{N}$, где $v:=\rho_{\mu}^{1 / 2}$. Для любого $m \in \mathbf{N}$ имеются локально-аналитические функ-

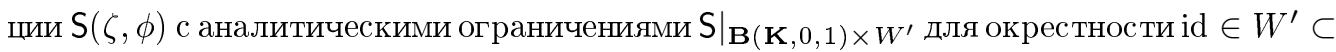
$W \cap G^{\prime}$ и условие $\operatorname{det}(\Psi(g))=0$ определяет аналитическое подмногообразие $G_{\Psi}$ в $G$ с $\operatorname{codim}_{\mathbf{K}} G_{\Psi} \geqslant 1$, где $\Psi(g)$ - матрища, зависящая от $g \in G$ с элементами $\Psi_{l, j}(g):=$ $P D_{c}\left(l, v\left(\mathrm{~S}\left(\zeta, \phi_{j}\right), g\right)\right)$ при $l \geqslant 1$. Если $f \in H$ такова, что $(f(g), v(\phi, g))_{H}=0$ для любых $\phi \in G^{\prime} \cap W$, то $P D_{c}\left(l,\left(f(g), v\left(\mathrm{~S}\left(\zeta, \phi_{j}\right), g\right)\right)_{H}\right)=0$. Тогда $f=0$, т.к. для любых $m \in \mathbf{N}$ имеются $\mathrm{S}\left(\zeta, \phi_{j}\right)$ с $\operatorname{det}(\Psi(g)) \neq 0 \quad \mu$-почти всюду на $G$, следовательно, вектор $f_{0}$ цикличен, где $f_{0}(g)=1$ для любого $g \in G$. Из выбора $V_{j}, V^{\prime}{ }_{j}$ в п. 3.6 и построения меры $\mu$ следует, что не сушествует ненулевого конечномерного $G^{\prime}$-инвариантного подпространства $H^{\prime}$ в $H$.

Пусть $\alpha_{F}(f)=F f$ для $F \in L^{\infty}(G, \mu, \mathbf{C})$ при любых $f \in H$. Пусть А $G$ обозначает *-подалгебру, порожденную семейством унитарных операторов $\left\{T_{h}: h \in G^{\prime}\right\}$ в алгебpe $\tilde{L}(H)$ непрерывных линейных операторов $B: H \rightarrow H$. В силу теоремы фон Неймана (см. $\S$ VI.24.2 из [14]) $\mathrm{A}_{G}^{\prime \prime}:=\left(\mathrm{A}_{G}^{\prime}\right)^{\prime}$ совпадает со слабым замыканием $\mathrm{A}_{G}$ в $\tilde{L}(H)$, где $\mathrm{A}_{G}^{\prime}$ обозначает коммутантную алгебру для $\mathrm{A}_{G}$. Пусть $\lambda$ является вероятностной действительной мерой на $G^{\prime} \operatorname{c} \operatorname{supp}(\lambda)=G^{\prime}$. Каждый оператор $\alpha_{F}$ принадлежит $\mathrm{A}_{G}^{\prime \prime}$, т.к. в силу цикличности $f_{0}$ существует функция $\beta(h)$, удовлетворяющая равенствам

$$
\begin{gathered}
\left(f_{s}, F f_{l}\right)_{H}=\int_{G} \int_{G^{\prime}} \bar{f}_{s}(g) \beta(h)\left(T_{h} f_{l}(g)\right) \lambda(d h) \mu(d g) \\
\int_{G} \bar{f}_{s}(g) F(g) f_{l}(g) \mu(d g)=\int_{G} \int_{G^{\prime}} \bar{f}_{s}(g) \beta(h) f_{l}(g) \lambda(d h) \mu(d g) .
\end{gathered}
$$

Если $\bar{p}$ является проектором на замкнутое $T$-инвариантное подпространство в $H$, то в соответствии с $\S$ VIII.19.2;5 работы [14] либо $\bar{p}=0$, либо $\bar{p}=I$.

\section{4. ПРИМЕНЕНИЕ ДИФФЕОМОРФИЗМОВ В КВАНТОВОЙ МЕХАНИКЕ И ТЕОРИИ ГРАВИТАЦИИ}

В качестве пространства-времени Минковского используется многообразие $M=\mathbf{Q}_{p}^{4}$ с билинейной функцией $\phi(x, y):=c^{2} x^{0} y^{0}-x^{1} y^{1}-x^{2} y^{2}-x^{3} y^{3}$, где $c$ - скорость света, 
$x=\left(x^{0}, x^{1}, x^{2}, x^{3}\right), x^{j} \in \mathbf{Q}_{p}$ [2]. В обшем случае можно рассмотреть БМ $M$, моделируемое на $\mathbf{K}^{n}$ или $c_{0}\left(\omega_{0}, \mathbf{K}\right)$. Зададим для него $p$-адический аналог интервала $d s^{2}:=$ $g_{i, j} d x^{i} d x^{j}$, где $\left(g_{i, j}\right)_{x}=g_{i, j}=g_{j, i}$ является локально-аналитической функцией по $x \in$ $M$; зададим также символы Кристоффеля $\Gamma_{i, j}^{k}$, тензор кривизны $R_{j, k, l}^{i}$, тензор Риччи $R_{i, j}=R_{i, l, j}^{l}$ и скалярную кривизну $R=R_{i, j} g^{i, j}$, где $g^{i, j} g_{j, k}=\delta_{k}^{i}$. Уравнения Эйнштейна гравитационного поля имеют вид $R_{i, j}-R g_{i, j} / 2+\lambda g_{i, j}=\kappa T_{i, j}$, где $T_{i, j}$ - тензор энергии-импульса [2].

Поскольку $G^{\prime}(\infty, M)$ плотно в $G^{\prime}(t, M)$, ограничение представления $T$ из п. 3.7 на $G^{\prime}(\infty, M)$ неприводимо. Согласно теореме 2 из работы [6] (см. также §3.1 из [8]) при $t^{\prime} \geqslant$ 1 в группе $G\left(t^{\prime}, M\right)$ сушествует открыто-замкнутая подгруппа $W^{\prime}$, так что каждый элемент $g \in W^{\prime}$ лежит хотя бы на одной однопараметрической подгруппе $g^{z}$. Это означает, что $\left(\partial g^{z} / \partial z\right)=A g^{z}$ с $z \in \mathbf{B}(\mathbf{K}, 0,1), g^{1}=g$ и $g^{0}=\mathrm{id}$, хотя $W^{\prime}$ и $G(\infty, M)$ не являются группами Банаха-Ли, где $g^{z+y}=g^{z} \circ g^{y}$ для любых $z$ и $y \in \mathbf{B}(\mathbf{K}, 0,1), A=A(x)$ - векторное поле на $M$, т. е. $A(x)=A^{1}(x) \partial_{1}+A^{2}(x) \partial_{2}+\cdots$ с $A^{i} \in C\left(t^{\prime}, M \rightarrow \mathbf{K}\right)$ для любого $x \in M, g=g(x), M \subset \mathbf{K}^{n}$. Для $c_{0}\left(\omega_{0}, \mathbf{K}\right)$ вместо всего топологически сопряженного пространства $c_{0}\left(\omega_{0}, \mathbf{K}\right)^{*}$ рассмотрим подпространство $c_{0}\left(\omega_{0}, \mathbf{K}\right)$, разделяюшее точки в исходном пространстве. Возьмем в качестве $T^{*} M$ для бесконечномерного над $\mathbf{K}$ банахова многообразия $M$ подмногообразие кокасательного расслоения, изоморфное $T M$. Тогда можно рассмотреть форму Лиувилля $\theta_{l}:=p_{i}(l)\left(d q^{i}\right)_{l}$ на $2 n$-мерном банаховом многообразии $T^{*} M$, где $l \in T^{*} M,\left(q^{1}, q^{2}, \ldots ; p_{1}, p_{2}, \ldots\right)$ - локальные координаты для кокасательного (под)расслоения $T^{*} M$ в базисе $\left(\left(d q^{1}\right)_{l},\left(d q^{2}\right)_{l}, \ldots ;\left(d p_{1}\right)_{l},\left(d p_{2}\right)_{l}, \ldots\right)$ и выполняется формула $\left\langle\theta_{l}, v\right\rangle_{l}:=\left\langle l, \pi_{*} v\right\rangle_{\pi(l)}$ для любых $v \in T_{l}\left(T^{*} M\right)$, где $\pi: T^{*} M \rightarrow M$ - проекция, $\pi_{*}-$ дифференциал отображения $\pi, n \leqslant \omega_{0}$. Банахово многообразие $T^{*} M$ является симплектическим, т.к. на нем сушествует замкнутая 2-форма $\sigma=-d \theta=d q^{1} \wedge$ $d p_{1}+d q^{2} \wedge d p_{2}+\cdots$. В работе [8] было доказано, что симплектическая группа $\operatorname{Sp}(2 n, \mathbf{K})$ над полем $\mathbf{K}$ вкладывается в $G(\infty, M)$ для любого $n \in \mathbf{N}$, в чем можно также убедиться, рассматривая $\psi \in G(\infty, M)$ с $\psi^{*} \sigma=\sigma$, где $\psi^{*}$ - операция ограничения тензорного поля, $\psi^{*}=\left(\psi_{*}\right)^{-1}$. Тогда каждый элемент $\phi \in G(\infty, M)$ сохраняет форму Лиувилля $\theta$, т.к. $\phi^{*}=: \psi \in G\left(\infty, T^{*} M\right), \psi^{*} \theta=\theta, \psi^{*} \sigma=\sigma$ согласно $\S 4$ из работы [1].

Пусть $C(\infty, M \rightarrow \mathbf{K})$ - абелева группа относительно поточечного сложения функций, элементы $h \in C(\infty, M \rightarrow \mathbf{K})$ действуют на локальные симплектические координаты преобразованием $\Psi_{h}(q, p)=\left(q,\left\{p_{j}-\partial h(q) / \partial q^{j}: j\right\}\right)$, где $q=\left(q^{1}, q^{2}, \ldots\right)$ и $p=$ $\left(p_{1}, p_{2}, \ldots\right)$. Каждый элемент $\phi \in G(\infty, M)$ задает преобразование $\tau_{\phi}(l):=\left(\phi^{-1}\right)^{*}(l)$, где $l \in T_{q}^{*} M$. Так же как в работе [1], полупрямое произведение групп $C(\infty, \mathbf{K}) \otimes^{s}$ $G(\infty, M)$ действует транзитивно и симплектически на $T^{*} M$.

Пусть $\gamma^{A}$ обозначает векторное поле на $T^{*} M$, ассоциированное с однопараметрической подгруппой диффеоморфизмов, соответствуюшей векторному полю $A$ на $M$. Тогда $\sigma\left(\gamma^{A}, B\right)=B\left\langle\theta, \gamma^{A}\right\rangle-\left\langle L_{\gamma^{A}}(\theta), B\right\rangle$ для любого векторного поля $B$ на $T^{*} M$, где $L_{\gamma^{A}}$ обозначает производную Ли для любой гладкой дифференциальной формы $w$ степени $l$ на БМ $N$. Действие $G(\infty, M)$ на $T^{*} M$ сохраняет форму Лиувилля $\theta$, поэтому $L_{\gamma^{A}} \theta=0$, следовательно, $\sigma\left(\gamma^{A}, B\right)=\left\langle d\left(\left\langle\theta, \gamma^{A}\right\rangle\right), B\right\rangle$, т. е. $\gamma^{A}$ является гамильтонианом.

Пространство метрик на БМ $\Sigma$ можно отождествить с $C(\infty, \Sigma \rightarrow S)$ при $\operatorname{dim}_{\mathrm{K}} \Sigma=$ 
$n \in \mathbf{N}$, где $S:=G L(n, \mathbf{K}) / O(n, \mathbf{K})$. При квантовании в теории гравитации можно взять $n=3$ и задать неотрицательную меру Хаара на $\mathbf{K}^{n}$ и индуцируемую ею дифференциальную форму $\theta_{x}:=(n !)^{-1} \epsilon_{j_{1}, \ldots, j_{n}}\left(\theta^{j_{1}} \wedge \theta^{j_{2}} \wedge \cdots \wedge \theta^{j_{n}}\right)_{x}$, где $\theta^{i}$ соответствуют 1-формам на $\mathbf{K}_{i}=\mathbf{K}, \epsilon$ - антисимметричный тензор с $\epsilon_{1,2, \ldots, n}=1$. Возьмем в качестве БМ $M=$ $C(l, \Sigma \rightarrow G L(n, \mathbf{K}))$ с $2<l<\infty$, где $\Sigma$ - открыто-замкнутое подмножество в $\mathbf{K}^{n}$. Тогда при рассмотрении хорошо известных уравнений Дирака и Вилера-Де Витта (см., например, $[1$, уравнения (6.3)-(6.12)]) квантование может быть сведено к нахождению представлений группы $G^{\prime}(\infty, M) \subset G(\infty, M)$.

Благодарности. Автор искренне благодарен И.В. Воловичу за обсуждение статьи.

\section{Список литературы}

[1] C. J. Isham. Topological and global aspects of quantum theory. In: Relativity, Groups and Topology. II. Ed. B. S. De Witt. Amsterdam: North-Holland, 1984. P. 1059.

[2] I. Ya. Aref'eva, B. Dragovich, P. H. Frampton, I. V. Volovich. Int. J. Mod. Phys. 1991. V. 6. P. 4341.

[3] В. С. Владимиров, И. В. Волович, Е.И. Зеленов. p-Адический анализ и математическая физика. М.: Физ.-мат. лит., Наука, 1994.

[4] A. C. M. van Rooij. Non-Archimedean Functional Analysis. New York: Marcel Dekker Inc., 1978.

[5] S. V. Ludkovsky. Southeast Asian Bull. Math. 1998. V. 22. P. 419.

[6] С. В. Людковский. УМН. 1996. Т. 51. № 2. С. 169.

[7] С. В. Людковский. УМН. 1996. Т. 53. № 3. С. 203.

[8] S. V. Ludkovsky. Representations and structure of groups of diffeomorphisms of non-Archimedean Banach manifolds. I, II. Preprints № IC/96/180, 181. Trieste: Int. Cent. Theor. Phys., 1996; http://www.ictp.trieste.it.

[9] Е. Т. Шавгулидзе. ДАН СССР. 1988. Т. 303. № 4. С. 811.

[10] W. H. Schikhof. Ultrametric calculus. Cambridge: Cambridge Univ. Press, 1984.

[11] Ю. Л. Далеикий, С. В. Фомин. Меры и диффференциальные уравнения в бесконечномерном пространстве. М.: Наука, 1983.

[12] B. С. Владимиров. УМН. 1989. Т. 43. № 5. С. 17.

[13] S. V. Ludkovsky. Quasi-invariant and pseudo-differentiable measures on a non-Archimedean Banach space. Preprint IC/96/210. Trieste: Int. Cent. Theor. Phys., 1996; № 3353-B97, 17.11.1997; М.: ВИНИТИ, 1997.

[14] J. M. G. Fell, R.S. Doran. Representations of *-Algebras, Locally Compact Groups, and Banach *-Algebraic Bundles. Boston: Acad. Press, 1988.

Поступила в редакцию 18.VI. 1998 г., после доработки 10.II. 1999 г. 